\title{
On the Full Transitivity of a Cotorsion Hull of the Pierce Group
}

\author{
Tariel Kemoklidze \\ Department of Mathematics, Akaki Tsereteli State University, Kutaisi, Georgia \\ Email: kemoklidze@gmail.com \\ Received 13 February 2014; revised 13 March 2014; accepted 18 March 2014 \\ Copyright (C) 2014 by author and Scientific Research Publishing Inc. \\ This work is licensed under the Creative Commons Attribution International License (CC BY). \\ http://creativecommons.org/licenses/by/4.0/

(c) (i) Open Access

\section{Abstract}

The paper considers the problem of full transitivity of a cotorsion hull $G^{\bullet}$ of a separable primary group $G$ when a ring of endomorphisms $E(G)$ of the group $G$ has the form $E_{s}(G) \oplus J_{p}$, where $E_{s}(G)$ is a subring of small endomorphisms of the ring $E(G)$, whereas $J_{p}$ is a ring of integer $p$-adic numbers. Investigation of the issue of full transitivity of a group is essentially helpful in studying its fully invariant subgroups as well as the lattice formed by these subgroups. It is proved that in the considered case, the cotorsion hull is not fully transitive. A lemma is proposed, which can be used in the study of full transitivity of a group and in other cases.

\section{Keywords}

Full Transitivity of a Group; Cotorsion Hull; Fully Invariant Subgroup

\section{Introduction}

The groups discussed in the paper are abelian and the operation is written in additive terms. We use here the notation and terminology of the monographs [1] [2].

The symbol $p$ denotes a fixed prime number. $Z$ and $Q$ are respectively the groups of integer and rational numbers. A subgroup $B$ of the group $A$ is called fully invariant if it is self-mapped for any endomorphism of the group $A$.

The knowledge of the construction of fully invariant subgroups of an abelian group and their lattice is essentially helpful in the study of the properties of the group itself and also in the investigation of the properties of its rings of endomorphisms and quasi-endomorphisms, the group of automorphisms and other algebraic systems connected with the initial group.

For a sufficiently wide class of $p$-groups these topics were studied by R. Baer, I. Kaplansky, P. Linton, R. 
Pierce, D. Moore, E. Hewett and others. The works of A. Mader, R. Göbel, P. A. Krylov, S. Ya. Grinshpon, A. I. Moskalenko and other authors are dedicated to the investigation of these topics in torsion-free and mixed groups.

However little is known about the results obtained in this area for the class of cotorsion groups. A group $A$ is called a cotorsion group if its extension by means of any torsion-free group $C$ splits as follows: $\operatorname{Ext}(C, A)=0$. The importance of the class of cotorsion groups in the theory of abelian groups is due to two factors: for any groups $A, B$, the group $\operatorname{Ext}(A, B)$ is a cotorsion one and any reduced group $G$ is isomorphically embeddable in the group $G^{\bullet}=\operatorname{Ext}(Q / Z, G)$ called the cotorsion hull of the group $G$. If the torsion part of the group $G$ is denoted by $t G$, then

$$
\operatorname{Ext}(Q / Z, t G) \cong \prod_{p} \operatorname{Ext}\left(Z\left(p^{\infty}\right), T_{p}\right)
$$

where $t G=\oplus T_{p}$. Thus the study of cotorsion groups essentially reduces to the study of groups of the form $\operatorname{Ext}\left(Z\left(p^{\infty}\right), T\right)=T^{\bullet}$, where $T$ is a $p$-primary group.

It is noteworthy that endomorpohisms in cotorsion groups are completely defined by their action on the torsion part and, as shown by W. May and E. Toubassi [3], for a mixed group $A$ the ring of endomorphisms $E(A)$ is isomorphic to $E(t A)$ if and only if $A$ is a fully invariant subgroup of the cotorsion hull $(t A)^{\circ}$.

The notion of full transitivity of a group plays an essential role in describing the lattice of fully invariant subgroups.

By the $p$-indicator of an element $a$ of the group $A$ we mean an increasing sequence of ordinal numbers

$$
H(a)=\left(h(a), h(p a), \cdots, h\left(p^{n} a\right), \cdots\right)
$$

where $h$ is the generalized $p$-height of an element, i.e. for $h(a)=\sigma$ if $a \in p^{\sigma} A \backslash p^{\sigma+1} A$ and $h(0)=\infty$. Now for the set of indicators we can introduce the order

$$
H(a) \leq H(b) \Leftrightarrow h\left(p^{i} a\right) \leq h\left(p^{i} b\right), i=0,1, \cdots
$$

A reduced $p$-group is called fully transitive if for arbitrary elements $a$ and $b$, when $H(a) \leq H(b)$ there exists an endomorphism $\varphi$ of the group such that $\varphi a=b$. The class of fully transitive groups includes such important groups as separable $p$-groups, algebraically compact groups and quasi-pure injective groups.

Using the indicators of fully transitive groups we can describe the lattice of fully invariant subgroups (see [4]-[11]).

For a module over a commutative ring, A. Mader formulated a general scheme that can be used to describe the lattice of fully invariant submodules of the module (see [10], Theorem 2.1 or [12], Theorem 1.1).

In the same way as we did for a $p$-group we define the notion of full transitivity for the group

$T^{\bullet}=\operatorname{Ext}\left(Z\left(p^{\infty}\right), T\right)$. According to A. Mader [10], an algebraically compact group is fully transitive and described with the aid of indicators the lattice of fully invariant subgroups of this group. This means to describe the lattice of fully invariant subgroups of the group $T^{\bullet}=\operatorname{Ext}\left(Z\left(p^{\infty}\right), T\right)$ when $T$ is a torsion-complete group. When $T$ is the direct sum of cyclic $p$-groups, A. Moskalenko [11] proved that $T^{\bullet}$ is also fully transitive and described by means of indicators the lattice of fully invariant subgroups of the group $T^{\bullet}$. In general, for the separable primary group $T$, the cotorsion hull $T^{*}$ is not fully transitive. In particular if $T$ is an infinite direct sum of torsion-complete groups, then, as shown by the author [13], the group $T^{\bullet}$ is not fully transitive and in that case the lattice of fully invariant subgroups of the group $T^{\bullet}$ cannot be described by means of indicators (see [12]).

R. Pierce [14] considered the primary group $G$, a ring of whose endomorphisms has the form

$$
E(G)=E_{s}(G) \oplus J_{p}
$$

where $E_{s}(G)$ is the ring of small endomorphisms of the group $G$ which is the ideal of the ring of endomor- 
phisms $E(G)$ of the group $G$, whereas $J_{p}$ is the ring of integer $p$-adic numbers. A small endomorphism of the group $G$ is defined as follows (see [14]).

For all $k \geq 0$ there exists an integer $n$ such that

$$
e(x) \leq k \text { and } h(x) \geq n \text { imply } \varphi(x)=0 .
$$

The Pierce group $G$ is important when studying the ring of endomorphisms of abelian groups (see [15]). The aim of the present paper consists in elucidating the full transitivity of the cotorsion hull $G^{*}$ and also in finding the conditions, under which the cotorsion hull is not fully transitive.

\section{Full Transitivity of the Cotorsion Hull of the Pierce Group}

As mentioned above, R. Pierce [14] considered the separable primary group $G$ with a standard basic subgroup $B=\bigoplus_{n=1}^{\infty} B_{n}, \quad B_{n} \cong Z\left(p^{n}\right), \quad n=1,2, \cdots, \quad B \subset G \subset \bar{B}$, where $\bar{B}$ is a torsion-complete group, i.e. the torsion part of a $p$-adic completion of the group $B$. The cardinality is $|G|=2^{\aleph_{0}}$ and the ring of endomorphisms of the group $G$ has form (1.1).

To study the full transitivity of the group $G^{*}$, we use the following representation of elements of the cotorsion hull of $T^{\bullet}$ given by A. Moskalenko [11] for the separable $p$-group $T$

$$
T^{\bullet}=\left\{\left(a_{0}, a_{1}+T, \cdots, a_{i}+T, \cdots\right) \mid a_{i} \in \hat{T}, p a_{i+1}-a_{i} \in T, i=0,1, \cdots\right\} .
$$

Representation of elements in this form makes it easy to calculate the height and the indicator. In particular, if $a=\left(a_{0}, a_{1}+T, \cdots\right)$, then

$$
H_{T^{*}}(a)==\left\{\begin{aligned}
H_{\hat{T}}\left(a_{0}\right) \quad & \text { if } \mathcal{O}\left(a_{0}\right)=\infty, \\
\left(h_{\hat{T}}\left(a_{0}\right), h_{\hat{T}}\left(p a_{0}\right), \cdots, h_{\hat{T}}\left(p^{n-1} a_{0}\right), \omega+m, \omega+m+1, \cdots\right) & \quad \text { if } a_{0} \in \hat{T} \backslash T, \mathcal{O}\left(a_{0}\right)=p^{n}, \mathcal{O}\left(a_{0}+T\right)=p^{n-m}, \\
\left(h_{T}\left(a_{0}\right), h_{T}\left(p a_{0}\right), \cdots, h_{T}\left(p^{n-1} a_{0}\right), \omega+n+k, \omega+n+k+1, \cdots\right) & \\
& \text { if } \mathcal{O}\left(a_{0}\right)=p^{n}, a_{0}, a_{1}, \cdots, a_{k} \in T, a_{k+1} \notin T, \\
H_{T}\left(a_{0}\right) & \text { if } a_{i} \in T \text { for any } i,
\end{aligned}\right.
$$

where $\omega$ is the smallest infinite ordinal number.

Let $B$ be a basic subgroup of the reduced separable $p$-group $T$ lying between $B$ and $\bar{B}$. Elements $a_{0}, b_{0} \in \hat{T}, \mathcal{O}\left(a_{0}\right)=\mathcal{O}\left(a_{0}\right)=p, a_{0} \notin T$. As is know, an endomorphism $\varphi$ of the group $T$ extends uniquely to an endomorphism of $\hat{T}$.

The following lemma is true.

Lemma 2.1. If $h_{\hat{T}}\left(a_{0}\right) \leq h_{\hat{T}}\left(b_{0}\right)$ and there exists no endomorphism $\varphi$ of the group $T$ for which $\varphi a_{0}=b_{0}$, then a cotorsion hull $T^{\bullet}$ is not fully transitive.

Proof. Consider two elements

$$
a=\left(a_{0}, a_{1}+T, a_{2}+T, \cdots\right), \quad b=\left(b_{0}, b_{1}+T, b_{2}+T, \cdots\right)
$$

of the group $T^{\bullet}$. Then by the condition of the theorem and (2.2) we have $H_{T^{*}}(a) \leq H_{T^{*}}(b)$. As is known, each endomorphism of the group $T$ extends uniquely to an endomorphism of the group $T^{\bullet}$. We will show that if for an endomorphism $\varphi, \varphi a=b$, then $\varphi a_{0}=b_{0}$. Let

$$
E: 0 \longrightarrow T \stackrel{\mu}{\longrightarrow} G \stackrel{\gamma}{\longrightarrow} \mathbb{Z}\left(p^{\infty}\right) \longrightarrow 0
$$

be the element of the group $T^{\bullet}$ defined by the sequence $a=\left(a_{0}, a_{1}+T, \cdots\right)$. For an endomorphism $\varphi$ of the group $T$ let us show that $\varphi a=\left(\varphi a_{0}, \varphi a_{1}+T, \cdots\right)$. According to ([1], Section 50), the extension of $\varphi E$ is 
defined from the commutative diagram

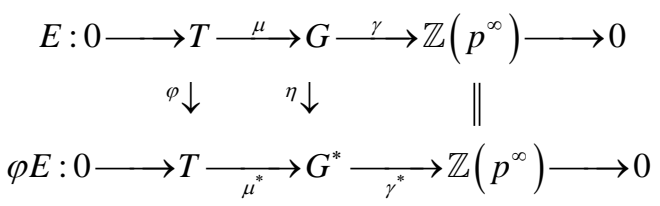

where $\mu$ is the identical inclusion,

$$
\begin{aligned}
& G^{*}=(T \oplus G) / H, \quad H=\{(-\varphi t, \mu t) \quad t \in T \mid\}, \\
& \mu^{*}: t \rightarrow(t, 0)+H, \quad \eta: g \rightarrow(0, g)+H, \\
& \gamma^{*}:(t, g)+H \rightarrow \gamma g, \quad t \in T, \quad g \in G .
\end{aligned}
$$

The commutativity of diagram (2.4) immediately follows from the definition of these homomorphisms.

To extension (2.3) there corresponds the sequence $\left(a_{0}, a_{1}+T, \cdots\right)$, where elements are defined as follows: fix a system of generators $\left\{\bar{g}_{n}: n \in N\right\}$ of the group $\mathbb{Z}\left(p^{\infty}\right), \bar{g}_{0}=0, p \bar{g}_{n+1}=\bar{g}_{n}$. Let $\left\{g_{n}\right\}, g_{0}=0$, be a system of representatives of the adjacent classes $\bar{g}_{n}$ of the group $G, \bar{g}_{n}=g_{n}+T, p g_{n+1}=g_{n}+c_{n}, c_{n} \in T$. Denote

$$
a_{i}=\lim _{n \rightarrow \infty}\left(c_{i}+p c_{i+1}+\cdots+p^{n} c_{i+n}\right), a_{i} \in \hat{T}, i=0,1, \cdots
$$

Then for each $i$,

$$
p a_{i+1}-a_{i}=-c_{i} \in T .
$$

For an endomorphism $\varphi$ of the group $T$ we have $\varphi c_{n} \in T, n \in N$, and can define

$$
\varphi a_{i}=\lim _{n \rightarrow \infty}\left(\varphi c_{i}+p \varphi c_{i+1}+\cdots+p^{n} \varphi c_{i+n}\right)
$$

It is obvious that the right-hand part of equality (2.5) defines the extension of an endomorphism $\varphi$ on $\hat{T}$ and if $\varphi^{*}$ is some other endomorphism of the group $\hat{T}$, which induces $\varphi$ on $T$, then $\operatorname{Ker}\left(\varphi-\varphi^{*}\right)$ contains $T \supset B$ and $\varphi=\varphi^{*}$ ([1], Proposition 34.1). From (2.5) we have

$$
p \varphi a_{i+1}-\varphi a_{i}=-\varphi c_{i} \in T .
$$

Now we can consider an element

$$
\left(\varphi a_{0}, \varphi a_{1}+T, \varphi a_{2}+T, \cdots\right)
$$

of the group $T^{\bullet}$ and with its aid define the corresponding short exact sequence.

Let $G^{\prime}$ be the group defined by a system of generators $T \cup\left\{g_{i}^{\prime}, i \in N\right\}$ which are defined by the relations of the group $T$ and the equalities $p g_{i+1}^{\prime}=g_{i}^{\prime}+\varphi c_{i}, \quad g_{0}^{\prime}=0, \quad i=1,2, \cdots$. Then

$$
E^{\prime}: 0 \longrightarrow T \stackrel{\mu^{\prime}}{\longrightarrow} G^{\prime} \stackrel{\gamma^{\prime}}{\longrightarrow} \mathbb{Z}\left(p^{\infty}\right) \longrightarrow 0
$$

where $\mu^{\prime}$ is the identical inclusion and, for each element $c+k g_{i}^{\prime}, \quad c \in T, k \in Z, \gamma^{\prime}\left(c+k g_{i}^{\prime}\right)=k \bar{g}_{i}^{\prime}$ is a short exact sequence. To extension (2.7) there corresponds sequence (2.6) (see [11], Proof of Theorem 1). Let us show that by using extensions (2.3) and (2.7) we can compose the commutative diagram

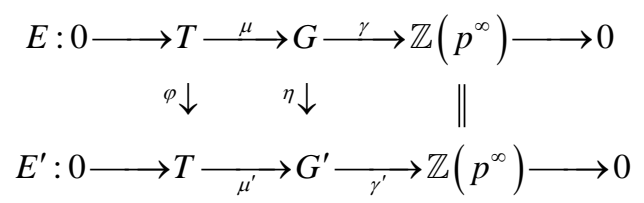

where $\varphi$ is the above-mentioned endomorphism and $\eta^{\prime}\left(c+k g_{i}\right)=\varphi c+k g_{i}^{\prime}, \quad c \in T, k \in Z, g_{i} \in G$. Indeed, from the definition of a triple $\left(\varphi, \eta^{\prime},=\right)$ we immediately conclude that (2.8) is a commutative diagram. 
Thus we have shown that (2.4) and (2.8) are commutative diagrams. Then, according to ([1], Section 50), $\varphi E$ and $E^{\prime}$ are equivalent extensions and thereby define one and the same sequence from $T^{\bullet}$. But, by virtue of our construction, $\left(\varphi a_{0}, \varphi a_{1}+T, \cdots\right)$ is the sequence corresponding to the extension $E^{\prime}$; therefore it corresponds to the extension $\varphi E$, too. Thus $\varphi a=\left(\varphi a_{0}, \varphi a_{1}+T, \cdots\right)$. Therefore if the endomorphism $\varphi$ maps the element $a=\left(a_{0}, a_{1}+T, \cdots\right)$ into $b=\left(b_{0}, b_{1}+T, \cdots\right)$, then $\varphi a_{0}=b_{0}, \varphi a_{1}+T=b_{1}+T, \cdots$, i.e. we have proved more than what has been mentioned at the beginning of the proof of the lemma. Thus it obviously follows that if there exists no endomorphism $\varphi$ of the group $T$ for which $\varphi a_{0}=b_{0}$, then there exists no endomorphism $\varphi$ of the group $T^{\bullet}$ which maps the element $a$ into $b$, i.e. $T^{\bullet}$ is not fully transitive. The lemma is proved.

For the Pierce group $G$ the following statement is true.

Theorem 2.1. The cotorsion hull $G^{\bullet}$ of the group $G$ is not fully transitive.

Proof. We use representation (2.1) of cotorsion hull elements and assume that $a=\left(a_{0}, a_{1}+G, \cdots\right)$ and $c=\left(c_{0}, c_{1}+G, \cdots\right)$ are elements of the group $G^{*}$, where $\mathcal{O}\left(a_{0}\right)=\mathcal{O}\left(c_{0}\right)=p, a_{0}, c_{0} \in \bar{B} \backslash G$. By virtue of (11, Item 2), elements $a_{0}$ and $b_{0}$ can be written in the form

$$
\begin{aligned}
& a_{0}=b_{0}+p b_{1}+p^{2} b_{2}+\cdots, \\
& c_{0}=b_{0}^{\prime}+p b_{1}^{\prime}+p^{2} b_{2}^{\prime}+\cdots,
\end{aligned}
$$

where $b_{i}, b_{i}^{\prime} \in B_{i}, i=0,1, \cdots$. Since $B \subset G \subset \bar{B}$ and $B$ is infinite, taking into account ([14]: Lemma 15.1, Theorem 15.4) we can assume that

$$
a_{0}-c_{0} \notin G
$$

By (2.2) we have $H_{T^{*}}(a)=H_{T^{*}}(c)=(0, \omega, \omega, \cdots)$, i.e. the following condition is fulfilled

$$
H_{T^{*}}(a) \leq H_{T^{*}}(c) \text {. }
$$

Let $\varphi$ be an endomorphism of the group $G$. Using (1.1) we have $\varphi=\psi+\alpha$, where $\psi$ is a small endomorphism of the group $G$ and $\alpha$ is the $p$-adic number $\alpha=\alpha_{0}+\alpha_{1} p+\alpha_{2} p^{2}+\cdots\left(\alpha_{n}=0,1, \cdots, p-1\right)$. As is known ([1], Section 39), the endomorphism $\varphi$ uniquely extends to the endomorphism $\bar{\varphi}=\bar{\psi}+\bar{\alpha}$ of the group $\bar{B}$

$$
\begin{aligned}
\bar{\varphi} a_{0} & =(\bar{\psi}+\bar{\alpha}) a_{0}=\bar{\psi} a_{0}+\bar{\alpha} a_{0} \\
& =\bar{\psi}\left(b_{0}+p b_{1}+p^{2} b_{2}+\cdots\right)+\bar{\alpha}\left(b_{0}+p b_{1}+p^{2} b_{2}+\cdots\right) .
\end{aligned}
$$

Since

$$
\mathcal{O}\left(p^{i} b_{i}\right) \leq p, \quad i=0,1, \cdots
$$

and $\varphi$ is a small endomorphism of the group $G$ (see (1.2)), starting with some $k$ we have

$$
\bar{\psi}\left(p^{k} b_{k}\right)=\psi\left(p^{k} b_{k}\right)=0 .
$$

Therefore

$$
\begin{aligned}
& \bar{\psi} a_{0}=\psi\left(b_{0}+p b_{1}+p^{2} b_{2}+\cdots+p^{k-1} b_{k-1}\right)=g \in G, \\
& b_{0}+p b_{1}+p^{2} b_{2}+\cdots+p^{k-1} b_{k-1} \in B \subset G .
\end{aligned}
$$

On the other hand, from (2.10) we obtain

$$
\begin{aligned}
\bar{\alpha} a_{0} & =\left(\alpha_{0}+\alpha_{1} p+\alpha_{2} p^{2}+\cdots\right)\left(b_{0}+p b_{1}+\cdots\right) \\
& =\alpha_{0} b_{0}+\alpha_{0} p b_{1}+\cdots=\alpha_{0}\left(b_{0}+p b_{1}+p^{2} b_{2}+\cdots\right)=\alpha_{0} a_{0} .
\end{aligned}
$$

Therefore 


$$
\bar{\varphi} a_{0}=g+\alpha_{0} a_{0}, \quad g \in G
$$

But $g+\alpha_{0} a_{0} \neq c_{0}$ since $\mathcal{O}\left(a_{0}\right)=p, \alpha_{0} \in\{0,1, \cdots, p-1\}$, and in that case the equality $g+\alpha_{0} a_{0}=c_{0}$ would contradict condition (2.9). Therefore

$$
\bar{\varphi} a_{0} \neq c_{0} .
$$

Thus there exists no endomorphism $\varphi$ of the group $G$ which extends to the endomorphism $\bar{\varphi}$ of the group $\bar{B}$ and $\bar{\varphi} a_{0}=c_{0}$. Then from Lemma 1.1 it follows that Theorem 2.1 is valid.

Note that one more example of a separable primary group, the cotorsion hull of which is not fully transitive, can be found in ([11], item 3).

As mentioned above, if the separable primary group $T$ is a direct sum of cyclic $p$-groups or a cotorsioncomplete group, then the cotorsion hull $T^{\bullet}$ is fully transitive. In 1993, at Professor A. Fomin's seminar A. Moskalenko made a conjecture that $T^{\bullet}$ is fully transitive only in these two cases. The proved lemma and theorem may serve as a positive argument in favor of this conjecture.

\section{Acknowledgements}

This study was supported by the grant (ATSU-2013/44) of Akaki Tsereteli University.

\section{References}

[1] Fuchs, L. (1970) Infinite Abelian Groups. I. Pure and Applied Mathematics, Vol. 36, Academic Press, New York.

[2] Fuchs, L. (1973) Infinite Abelian Groups. II. Pure and Applied Mathematics, Vol. 36-II, Academic Press, New York.

[3] May, W. and Toubassi, E. (1976) Endomorphisms of Abelian Groups and the Theorem of Baer and Kaplansky. Journal of Algebra, 43, 1-13. http://dx.doi.org/10.1016/0021-8693(76)90139-3

[4] Baer, R. (1935) Type of Elements and Characteristic Subgroups of Abelian Groups. Proceedings of the London Mathematical Society, Series 2, 39, 481-514. http://dx.doi.org/10.1112/plms/s2-39.1.481

[5] Kaplansky, I. (1969) Infinite Abelian Groups. The University of Michigan Press, Ann Arbor.

[6] Linton, R.S. (1976) On Fully Invariant Subgroups of Primary Abelian Groups. Michigan Mathematical Journal, 22, 281-284. http://dx.doi.org/10.1307/mmj/1029001528

[7] Moore, J.D. and Hewett, E.J. (1971/72) On Fully Invariant Subgroups of Abelian p-Groups. Commentarii Mathematici Universitatis Sancti Pauli, 20, 97-106.

[8] Göbel, R. (1974) The Characteristic Subgroups of the Baer-Specker Group. Mathematische Zeitschrift, 140, $289-292$. http://dx.doi.org/10.1007/BF01214169

[9] Grinshpon, S.Ya. and Krylov, P.A. (2005) Fully Invariant Subgroups, Full Transitivity, and Homomorphism Groups of Abelian Groups. Journal of Mathematical Sciences, 128, 2894-2997. http://dx.doi.org/10.1007/s10958-005-0245-5

[10] Mader, A. (1970) The Fully Invariant Subgroups of Reduced Algebraically Compact Groups. Publicationes Mathematicae Debrecen, 17, 299-306.

[11] Moskalenko, A.I. (1989) Cotorsion Hull of a Separable Group. Algebra i Logika, 28, 207-226. http://dx.doi.org/10.1007/BF01979377

[12] Kemoklidze, T. (2013) The Lattice of Fully Invariant Subgroups of the Cotorsion Hull. Advances in Pure Mathematics, 3, 670-697. http://www.emis.de/journals/GMJ/vol13/contents.htm http://dx.doi.org/10.4236/apm.2013.38090

[13] Kemoklidze, T. (2006) On the Full Transitivity of a Cotorsion Hull. Georgian Mathematical Journal, 13, 79-84.

[14] Pierce, R.S. (1963) Homomorphisms of Primary Abelian Groups. In: Topics in Abelian Groups, Scott, Foresman and Co., Chicago, 215-310.

[15] Stringall, R.W. (1967) Endomorphism Rings of Primary Abelian Groups. Pacific Journal of Mathematics, 20, 535-557. http://dx.doi.org/10.2140/pjm.1967.20.535 